\title{
Ranking of fibre-reinforced composite plate surface finish quality by wavelet texture analysis
}

\author{
S Palmer, W Hall and J Summerscales
}

\begin{abstract}
In the automotive and other industries, the visual appearance of external surfaces is a key factor in perceived product quality. Traditionally, the quality of an automotive surface finish has been judged by expert human auditors. A set of 17 fibre-reinforced composite plates was previously manufactured to have a range of surface finish qualities and these plates were ranked by three expert observers and also optically digitally imaged. Following validation of the previous rankings, the wavelet texture analysis (WTA) technique was applied to the digital photographs to derive an instrumental measure of surface finish quality based on the panel images. The rank correlation between the human expert surface finish quality ratings and those from the WTA image analysis process was found to be positive, large and statistically significant. This finding indicates that WTA could form the basis of an inexpensive and practical instrumental method for the ranking of fibre-reinforced composite surface finish quality.
\end{abstract}

\section{Introduction}

In the automotive and other industries, it has long been realised that the visual appearance of external surfaces is a key factor in perceived product quality ${ }^{[1,2]}$. The now widespread use of fibrereinforced polymeric composites on external product surfaces means that the surface finish on composite panels is of critical importance for customer satisfaction ${ }^{[3]}$. However, it is only in the last decade that investigations into the surface quality of composite laminates have become common in the literature ${ }^{[4]}$. Traditionally, the quality of automotive surface finish has been judged by expert human auditors ${ }^{[1]}$. It has also been the most commonly used and reliable approach for evaluation of the surface quality of composites $^{[5]}$. While it is possible for an expert rater to make an absolute assessment of surface quality, it is also common for human assessors to undertake visual assessments by ranking a number of samples, either indirectly as a series of pair-wise comparisons or directly into an ordinal sequence ${ }^{[1,5,6]}$.

The use of human inspectors in assessing surface quality, while effective, is labour-intensive, does not lend itself to automation and can have problems with subjectivity in rating and inter-rater reliability ${ }^{[7]}$. However, many available objective instrumental techniques for the measurement of surface finish quality do not always correlate well with human assessment ${ }^{[1]}$; it has been suggested that the surface roughness parameters commonly used do not completely characterise the visually-perceived quality ${ }^{[5,8]}$. So, an objective instrumental technique that can provide comparable results to human experts is desirable ${ }^{[1,5]}$. ASTM E430-11 describes standard test methods for measurement of the gloss of highgloss surfaces by abridged goniophotometry and is used by the automotive industry, however the equipment required (for example a BYK-Gardner GmbH wave-scan Dual) costs more than US\$40k. The process by which humans quickly and accurately assess surface quality is not well understood ${ }^{[2]}$, though experiments have shown that the human visual cortex appears to perform a twodimensional multi-scale decomposition of the visual field into a range of frequency bands ${ }^{[9]}$. Previous work has attempted to apply this multi-channel filtering theory of human vision to the task of quantitative measurement of surface texture ${ }^{[10]}$. Later work noted the similarity of the multi-channel filtering operation of the wavelet transform to biological vision systems and the use of the twodimensional discrete wavelet transform (2DDWT) in biologicallyinspired image processing systems ${ }^{[11]}$.

Wavelet techniques have been successfully applied in many nondestructive testing applications for the detection of flaws, including using data derived from optical ${ }^{[12]}$ and radiographic ${ }^{[13]}$ testing methods. There are also many reported examples of the application of wavelet techniques in the characterisation of composite materials, for example the detection of internal defects via acoustic emission pulse data ${ }^{[14]}$. Specifically, it has been shown that the wavelet transform has the ability to effectively characterise surface profile data (obtained via stereoscopic scanning electron microscopy) that contain multi-scale features and are non-stationary (containing localised variations) $)^{[15]}$. For the comprehensive characterisation of surface features and texture, these inherent abilities of the wavelet transform place it 'way ahead of other traditional methods ${ }^{\text {' }}{ }^{[8]}$ and are why it is 'generally considered to be state-of-the-art in texture analysis ${ }^{\text {[16] }}$.

Previous work has established the general feasibility of wavelet texture analysis (WTA) for the task of automatically classifying the surface finish of both clear resin with plain weave and gel-coat carbon fibre-reinforced polymer panels into broad quality grades ${ }^{[17,18]}$ and that WTA will produce repeatable results if reasonable precautions are taken in sample imaging ${ }^{[19]}$. This paper demonstrates the application of WTA to the new task of automatically ranking the

\section{Submitted 01.08.15 / Accepted 25.04.16}

Dr Stuart Palmer is an Associate Professor in the Faculty of Science, Engineering and Built Environment at Deakin University in Australia. He has a Bachelor of Engineering with Distinction, a Master of Business Administration in Technology Management and a Doctor of Technology. He is a chartered professional engineer and a member of Engineers Australia.

Dr Wayne Hall BEng(Hons) PhD PGCert CEng MIMechE is a Senior Lecturer in Mechanical Engineering at Griffith University in Australia.

Dr John Summerscales CEng CEnv CSci BSc MSC PhD PGDipEd FIMMM FInstNDT FIAQP is an Associate Professor (Reader) in Composites Engineering at the University of Plymouth in the UK. 
surface finish quality of fibre-reinforced composite plates. A set of sample plates was previously ranked for surface finish by expert observers, wave-scan DOI and fractal dimension analysis of digital photographs, as described in Insight by Labrosse et al ${ }^{[20]}$. The new analysis and results presented here include validation of the previous plate image rankings, application of the WTA technique to the images to derive an instrumental measure of surface finish quality based on the panel images and confirmation of the rank correlation between the human expert surface finish quality ratings and those from the WTA image analysis process.

\section{Experimental procedure}

A series of fibre-reinforced plates was produced and a range of surface finishes was obtained by varying aspects of the manufacturing process. A panel of three expert observers individually ranked the quality of the surface finish of the plate set. The plates were imaged using a high-resolution digital camera in all four orientations/ rotations $\left(0^{\circ}, 90^{\circ}, 180^{\circ}\right.$ and $270^{\circ}$ in plane). The equipment costs (digital camera, light-proof box and personal computer) were less than US\$2000. The full details of this prior experimental work, including the plate materials, manufacture and dimensions, the digital imaging apparatus and ranking results, were described in an earlier issue of Insight ${ }^{[20]}$. All of the following numerical analyses were performed using the Matlab computing environment ${ }^{[21,22]}$. An assessment of the inter-rater reliability of the quality rankings was made based on Spearman's and Kendall's rank correlation coefficients. Spearman's rho $(\rho)$ provides a measure of how well the relationship between two variables can be described by a monotonic function; in this case, if all ranking pairs from the lowest to the highest increase together, then $\rho=1.0$. Kendall's tau $(\tau)$ provides a measure of the similarity of the ordering of two sets: if two sets have identical rank ordering, then $\tau=1.0$. A combined reference surface finish quality ranking was developed for the plate set. Wavelet texture analysis (WTA) was applied to the plate image set. The WTA process consists of four steps: (i) iterative decomposition of the sample images using the 2DDWT; (ii) construction of a texture feature vector for each sample image; (iii) texture dimensional reduction using principal component analysis (PCA); and (iv) visualisation of the samples in the reduced dimensional space. These steps are described in the following sections.

The 2DDWT decomposes an image into three sets of 'detail' coefficients that represent high-frequency information in the original image, with horizontal, vertical and diagonal $\left(c D_{n}^{h}, c D_{n}^{v}\right.$ and $c D_{n}^{d}$ ) orientations, respectively ${ }^{[12,13]}$. Additionally, it produces a set of 'approximation' coefficients $\left(c A_{n}\right)$, which represent the remaining lower-frequency information in the original image. All four sets of coefficients are half the linear dimensions of the original image. The resultant approximation coefficients can be successively used as the input for further 2DDWT decomposition until the final approximation coefficients are too small for further transformation. For the size of images available for this analysis, six levels of decomposition are generally all that is possible. This 'multi-resolution' analysis, as represented in Figure 1, decomposes the original image into essentially orthogonal sets of coefficients that separately represent all of the information in the original image at different scales/distances and orientations. The complete decomposition after any level $j$ is all of the detail coefficient sets developed to that point plus the final remaining approximation coefficients $\left(c A_{j}\right)$, ie those elements included in the shaded region in Figure 1. The final approximation coefficients typically represent only the background illumination in the original image and are

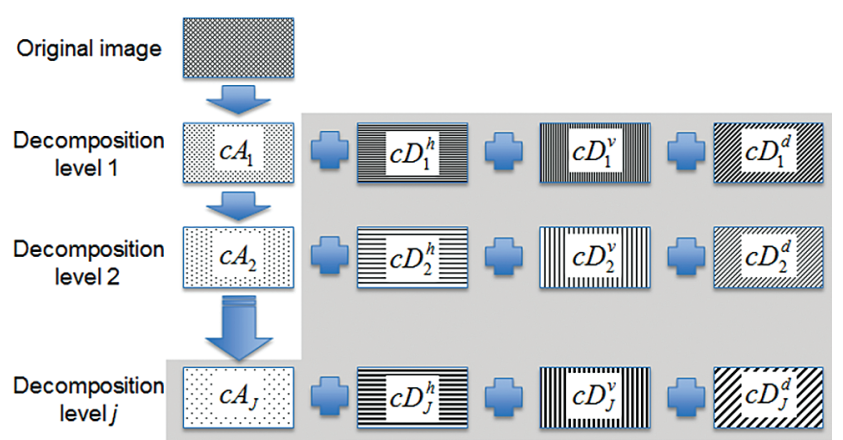

Figure 1. Multi-resolution image decomposition via the 2DDWT

generally not used in further analysis, and they are not used here. Wavelet techniques require the selection of a wavelet basis for the decomposition. One heuristic selection approach involves analysing sample data with a range of candidate wavelets and applying selection criteria to identify the optimal analysis wavelet ${ }^{[12,23]}$.

Rather than the raw detail coefficients, WTA collapses each set of coefficients into a single figure 'energy' measure. A range of energy measures is possible ${ }^{[12,16]}$; here, the square of the Frobenius norm of the wavelet detail coefficients, normalised by the size of the coefficient set, is used as the energy measure, as given in Equation (1):

$$
E_{j k}=\frac{1}{M \times N}\left\|c D_{j}^{k}\right\|_{F}^{2}(-J \leq j \leq 1 ; k=h, v, d) .
$$

where $j$ is the wavelet analysis scale/level, $J$ is the maximum desired analysis level, $k$ is the wavelet detail coefficient set orientation (horizontal, vertical or diagonal) and $M \times N$ is the size of the coefficient set. The square of the Frobenius norm of matrix A is defined as:

$$
\|A\|_{F}^{2}=\sum_{i, j}\left|a_{i j}\right|^{2}
$$

A texture feature vector for each plate image, containing $3 j$ elements, is assembled according to the form given in Equation (3):

$$
\left[E_{1 h}, E_{1 v}, E_{1 d}, E_{2 h}, E_{2 v}, E_{2 d}, \ldots E_{J h}, E_{J v}, E_{J d}\right]
$$

This feature vector is a rich representation of the texture in an image that includes features related to both scale and orientation. However, it typically contains a significant proportion of redundant information, with the actual determinants of differences in visual quality across the plate set confined to a limited range of scales and/or orientations. PCA is a standard statistical technique that transforms a set of correlated variables into a set of uncorrelated variables called 'principal components', with each being a linear combination of the original variables. The linear scaling factor computed for each of the original variables contributing to the value of a particular principal component is known as the loading coefficient for that variable in that principal component. The PCA transformation results in the first principal component containing as much of the variability in the original data as possible. Each successive principal component contains as much of the remaining variability as possible, while being orthogonal to all preceding principal components. In this way, the first few (and sometimes just the first) principal components embody most of the information in the original variables, effectively reducing the dimensionality of the original data significantly.

WTA incorporating six levels of wavelet multi-resolution decomposition was applied to each of the four rotation images for each sample plate. The corresponding elements in the four texture feature vectors for each sample plate were averaged to produce a single composite texture feature vector for each plate. PCA was 
applied to the set of all 17 plate texture feature vectors to explore the dimensionality of the image data. The locations of the 17 plates were mapped into the first two dimensions of the transformed principal component space to visually identify any obvious relationship between the plate principal component scores and the benchmark plate ranking. These steps were repeated for a range of common wavelet bases, with the aim of identifying the wavelet that maximised the explanatory power of the first few principal components resulting from the PCA stage. Once a candidate wavelet basis was identified, the level of agreement between the human expert surface finish quality ranking and the instrumentally-derived ranking from the WTA image analysis process was established based on Spearman's and Kendall's rank correlation coefficients. Related results and a discussion of all the findings are also presented.

\section{Results and discussion}

The results of the fibre-reinforced plate surface finish quality ranking were previously presented ${ }^{[20]}$ (see Figure 5 in the reference). Table 1 presents the detailed ranking results for the three expert observers (John Summerscales, Quentin Labrosse and Richard Cullen) and the variation (range) in ranking for each plate. Note that a lower ranking indicates a higher perceived level of surface finish quality. All observers agreed that there were a number of plates of a quality level that would not be acceptable in industrial applications; these are indicated in Table 1 by an asterisk $\left(^{*}\right)$ in the Plate ID column. Spearman's rho $(\rho)$ and Kendall's tau $(\tau)$ rank correlation coefficients were calculated for each rater pair as a measure of interrater reliability and the results are presented in Table 2 .

Table 1. Ranking results for plate surface finish quality from expert observers

\begin{tabular}{|c|c|c|c|c|c|c|}
\hline $\begin{array}{c}\text { Plate } \\
\text { ID }\end{array}$ & $\begin{array}{c}\text { Rank } \\
\text { JS }\end{array}$ & $\begin{array}{c}\text { Rank } \\
\text { QL }\end{array}$ & $\begin{array}{c}\text { Rank } \\
\text { RC }\end{array}$ & Range & $\begin{array}{c}\text { Rank } \\
\text { Av }\end{array}$ & $\begin{array}{c}\text { Combined } \\
\text { rank }\end{array}$ \\
\hline${ }^{*}$ & 16 & 13 & 16 & 3 & 15.00 & 15 \\
\hline 4.1 & 10 & 11 & 10 & 1 & 10.33 & 10 \\
\hline 4.2 & 5 & 6 & 7 & 2 & 6.00 & 6 \\
\hline 4.3 & 7 & 4 & 4 & 3 & 5.00 & 5 \\
\hline 5.1 & 2 & 2 & 1 & 1 & 1.67 & 2 \\
\hline 5.2 & 1 & 1 & 2 & 1 & 1.33 & 1 \\
\hline 6.2 & 9 & 9 & 8 & 1 & 8.67 & 8 \\
\hline 6.3 & 6 & 7 & 6 & 1 & 6.33 & 7 \\
\hline 77.1 & 15 & 17 & 14 & 3 & 15.33 & 16 \\
\hline$* 7.2$ & 13 & 14 & 13 & 1 & 13.33 & 13 \\
\hline$* 7.3$ & 14 & 15 & 15 & 1 & 14.67 & 14 \\
\hline$* 8.1$ & 17 & 16 & 17 & 1 & 16.67 & 17 \\
\hline 8.2 & 12 & 12 & 9 & 3 & 11.00 & $=11$ \\
\hline 8.3 & 8 & 8 & 11 & 3 & 9.00 & 9 \\
\hline 9.1 & 11 & 10 & 12 & 2 & 11.00 & $=11$ \\
\hline 9.2 & 3 & 5 & 5 & 2 & 4.33 & 4 \\
\hline 9.3 & 4 & 3 & 3 & 1 & 3.33 & 3 \\
\hline & & & & & & \\
\hline
\end{tabular}

The average variation in plate ranking was only 1.76 , which compares favourably to a similar multi-rater rank-based analysis ${ }^{[5]}$. All inter-rater rank correlation coefficients were positive, large and statistically significant. There was a high degree of agreement between the raters, suggesting that a simple mean of the three
Table 2. Inter-rater reliability measures for expert observers

\begin{tabular}{|c|c|c|c|c|} 
& \multicolumn{2}{c|}{ Spearman's rho } & \multicolumn{2}{c|}{ Kendall's tau } \\
\cline { 2 - 6 } & Rater QL & Rater RC & Rater QL & Rater RC \\
\hline \multirow{2}{*}{ Rater JS } & $\rho=0.958$ & $\rho=0.949$ & $\tau=0.868$ & $\tau=0.824$ \\
& $p<2 \times 10^{-9}$ & $p<7 \times 10^{-9}$ & $p<2 \times 10^{-6}$ & $p<4 \times 10^{-6}$ \\
\hline \multirow{2}{*}{ Rater QL } & - & $\rho=0.941$ & - & $\tau=0.809$ \\
& & $p<2 \times 10^{-8}$ & - & $p<6 \times 10^{-6}$ \\
\hline
\end{tabular}

rank scores for each plate can be used as the basis for an overall combined rank score, which when re-ranked then becomes the reference/benchmark rank, as used in the similar previous analysis noted above ${ }^{[5]}$.

Applying the WTA process to the 17 plate image set using a range of common wavelet bases showed that the first principal component from the PCA stage generally explained more than $90 \%$ of the variation in the information contained in the images. In particular, WTA based on a biorthogonal wavelet with order 2,2 (two vanishing moments for both the decomposition and reconstruction wavelet functions $)^{[11,22]}$ produced a first principal component accounting for $99.09 \%$ of the variation and was used to obtain the following results. Figure 2 shows the location of the 17 plates mapped in two dimensions using their first two principal component scores derived from the PCA stage. Noting that the second principal component scores are essentially irrelevant in this case (explaining only $0.56 \%$ of the image data variation), the rank ordering of the plates suggested by the WTA method is simply the sequence of plate scores from left to right (most negative to most positive) on the first principal component (horizontal) axis. Table 3 shows the plate rank ordering based on Figure 2 and also gives the corresponding benchmark combined ranking for each plate reproduced from Table 1. Figure 3 shows a scatterplot of the benchmark combined ranking from expert observers for each plate versus the rank ordering developed from the WTA method.

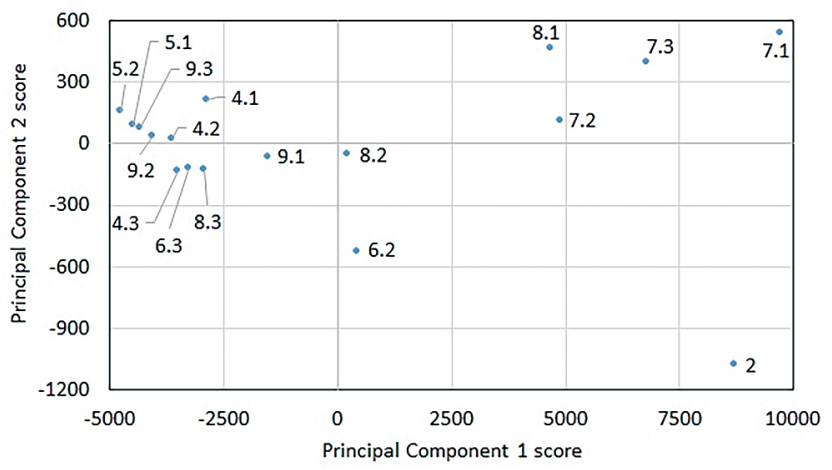

Figure 2. Plot of the first two principal components showing the location of all sample plates

Figure 3 suggests a strong association between the benchmark combined plate ranking from the expert observers and that derived by the WTA method. As noted in Tables 1 and 3, plates 8.2 and 9.1 were tied for eleventh position in the combined expert observer ranking. The WTA ranking is derived from the first principal component score, which is a continuous real variable and hence is unlikely to ever indicate a tied ranking. In this situation, the maximum possible values for rank correlation coefficients arising from a comparison of the combined expert observer ranking and the WTA ranking will be somewhat less than 1.0. For the results presented in Figure 3, Spearman's rho was $0.948\left(p<8 \times 10^{-9}\right)$ 
Table 3 . Ranking results for plate surface finish quality derived from the WTA method

\begin{tabular}{|c|c|c|c|}
\hline Plate ID & PC1 score & PC1 rank & Combined rank \\
\hline 2 & 8724.09 & 16 & 15 \\
\hline 4.1 & -2873.80 & 9 & 10 \\
\hline 4.2 & -3621.67 & 5 & 6 \\
\hline 4.3 & -3523.27 & 6 & 5 \\
\hline 5.1 & -4493.84 & 2 & 2 \\
\hline 5.2 & -4759.73 & 1 & 1 \\
\hline 6.2 & 417.02 & 12 & 8 \\
\hline 6.3 & -3279.06 & 7 & 7 \\
\hline 7.1 & 9717.64 & 17 & 16 \\
\hline 7.2 & 4880.63 & 14 & 13 \\
\hline 7.3 & 6779.14 & 15 & 14 \\
\hline 8.1 & 4672.35 & 13 & 17 \\
\hline 8.2 & 210.24 & 11 & $=11$ \\
\hline 8.3 & -2923.16 & 8 & 9 \\
\hline 9.1 & -1528.93 & 10 & $=11$ \\
\hline 9.2 & -4073.15 & 4 & 4 \\
\hline 9.3 & -4324.49 & 3 & 3 \\
\hline
\end{tabular}

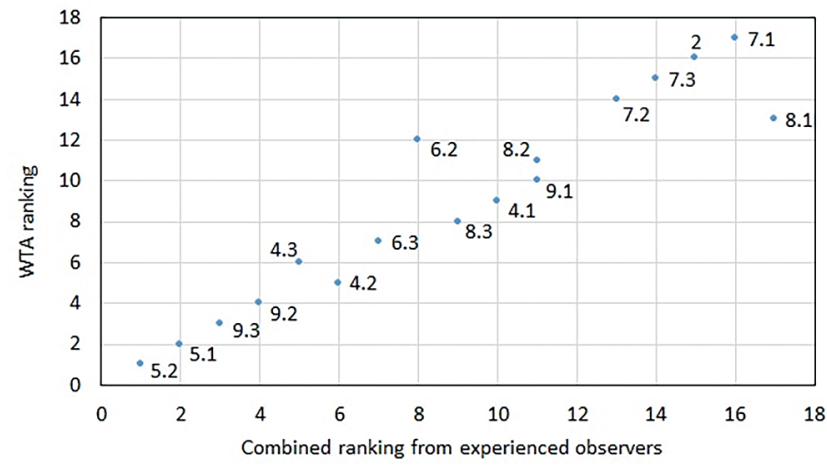

Figure 3. Benchmark combined ranking versus WTA ranking for sample plates

and Kendall's tau was $0.863\left(p<2 \times 10^{-6}\right)$. Both rank correlation coefficients were positive, large and statistically significant, supporting the view that there is good agreement between the human expert surface finish quality ranking and the instrumentallyderived ranking from the WTA image analysis process.

It can be seen that the five plates assessed by the expert observers as having a particularly poor surface finish quality (plates 2, 7.1, 7.2, 7.3 and 8.1 , indicated by an asterisk in Table 1) are also the plates given the lowest instrumental quality ratings (highest rankings) by the WTA method, see Table 3 and Figure 3. It can also be seen that these five plates are clearly separately grouped in the right half of Figure 2, confirming the ability of the WTA method to act as an accurate categorical classifier of composite surface finish quality ${ }^{[17,18]}$. Here, a simple good/bad quality classification criterion could be based on first principal component scores for sample plates as either less than/ greater than, say, 2500.
As previously noted, the PCA process transforms a set of variables into an equal number of principal components, which are linear combinations of the original variables, but with most of the information in the original variables concentrated in the first few principal components. The first principal component (PC1) score for a particular plate is the sum of all the products of each element in the texture feature vector for the plate and the corresponding PC1 loading coefficient derived in the PCA process. Table 4 shows the PC1 loading coefficients computed here via PCA for each element (level and orientation) of a plate texture feature vector.

PC1 is of particular importance here, as it was found to explain more than $99 \%$ of the variation in the image data across the set of all 17 plate images. Table 4 shows that the PC1 loading coefficients are very small until the fifth decomposition/analysis level and that it is the sixth level that makes the major contribution to the PC1 score. This indicates that it is features in the plate images contributing details/energy corresponding to the characteristic analysis length at level six that make the major contribution to the PC1 score for the plates. Hence, it is these features that are the main determinant of the variation in quality between the 17 sample plates in the WTA method used here. The characteristic length of the biorthogonal 2,2 wavelet used here is approximately $2^{l}$ pixels, where $l$ is the analysis level. Hence, at analysis level six, the characteristic length is approximately 64 pixels, which for the plate size and digital imaging configuration employed here equates to a size of approximately $7.5 \mathrm{~mm}$. A physical interpretation of this result is that, in the case presented here, it was the relative abundance of defect features in a size range centred around approximately $7.5 \mathrm{~mm}$ on the sample plates that was the primary determinant of the instrumentally-derived surface finish quality measure. The loading coefficients presented in Table 4 also indicate that surface features of $3.75 \mathrm{~mm}$ in size or less contribute very little to PC1 and hence ranking measurements based on PC1 will have a high level of immunity to measurement noise that might be introduced by the digital imaging process.

Figure 3 indicates two plates (6.2 and 8.1) that appear to depart from the otherwise regular rank relationship between the human observers and the instrumental WTA method. It is not immediately clear why plate 6.2 might be a relative outlier; however, an examination of the texture feature vectors, based on the biorthogonal 2,2 wavelet, for all 17 plates shows that plate 8.1 has the highest ratio of level five to level six energy measures, for both the horizontal and vertical orientations, of all plates. This suggests that plate 8.1 has a higher relative proportion of defect features centred on the level five characteristic length (here approximately $3.75 \mathrm{~mm}$ ) than the other plates. It further suggests that the human raters 'counted' these smaller defects in their overall subjective assessment of surface finish quality for plate 8.1. While these 'level 5' features have appeared in the texture feature vector for plate 8.1, the greater weighting given to level 6 features
Table 4. PC1 loading coefficients

\begin{tabular}{|c|c|c|c|c|c|c|}
\hline Level & Element & Loading & Element & Loading & Element & Loading \\
\hline 1 & Horizontal & $-2.34 \times 10^{-6}$ & Vertical & $-2.28 \times 10^{-6}$ & Diagonal & $-5.26 \times 10^{-7}$ \\
\hline 2 & Horizontal & $6.26 \times 10^{-6}$ & Vertical & $4.54 \times 10^{-7}$ & Diagonal & $-6.59 \times 10^{-6}$ \\
\hline 3 & Horizontal & 0.0007 & Vertical & 0.0004 & Diagonal & $-3.09 \times 10^{-6}$ \\
\hline 4 & Horizontal & 0.0179 & Vertical & 0.0132 & Diagonal & 0.0011 \\
\hline 5 & Horizontal & 0.1835 & Vertical & 0.2348 & Diagonal & 0.0585 \\
\hline 6 & Horizontal & 0.6340 & Vertical & 0.6322 & Diagonal & 0.3250 \\
\hline
\end{tabular}


in the PCA model developed as part of the WTA process means that their full impact on surface finish quality was not adequately accounted for in the instrumental quality rating. So, while Figure 3 indicates that the WTA model is generally in accord with the human assessments of surface finish quality, there appear to be some aspects not fully accounted for in the linear model developed via the WTA method.

It is important to note that the PCA procedure has no dependent or output variable(s) that represent the ideal plate ranking against which algorithmic error is minimised; the observed relationship between the PC1 score and the combined ranking from expert observers arises naturally from the PCA process, which seeks to load as much of the variability of the plate image data as possible into PC1. The imperfect relationship shown in Figure 3 suggests that it may be possible to develop a better model via a technique that explicitly seeks a goal rank ordering, such as ordinal regression. In a similar application (the rating of surface finish quality of textiles), it was shown that an artificial neural network (a form of non-linear classifier that employs a training cycle) was very effective at taking wavelet texture feature vector data (as used here) and producing a model that yielded ratings that closely agreed with human expert raters $^{[24]}$.

\section{Conclusions}

A set of 17 fibre-reinforced composite plates previously manufactured to have a range of surface finish quality, and which were previously ranked by three expert observers, were used as the basis for testing an instrumental method for surface finish quality ranking based on WTA. The inter-rater reliability of the original three expert observers was confirmed, providing the basis for a benchmark surface finish quality ranking. The WTA method was applied to images of the 17 plates using a range of common wavelet bases to maximise the variation in the plate images explained by the first principal component developed via PCA. Following the identification of the biorthogonal wavelet with order 2,2 as a desirable WTA basis, the PC1 scores thus obtained for the panels were used as an instrumental measure of surface finish quality and their rank order was compared to the benchmark human rater ranking. The rank correlation between the human expert surface finish quality ratings and those from the WTA image analysis process was found to be positive, large and statistically significant. This finding indicates that WTA could form the basis of a practical instrumental method for the ranking of fibre-reinforced composite surface finish quality with good agreement with human expert raters. A small number of significant discrepancies in the rank agreement between human and WTA ratings was observed, apparently due to a variation in the distribution of defect sizes between panels that is not well catered for by the 'linear combination of frequency band energies' structure inherent in the PCA model. This suggests possible fruitful areas for future research to further improve performance of the instrumental ranking method, including incorporating modelling approaches that feature output rank explicitly as an objective and/or non-linear modelling approaches such as artificial neural networks.

\section{Acknowledgements}

The authors thank Quentin Labrosse, Christopher Hoppins and Richard Cullen for their various respective contributions that supported the analysis presented here, including the preparation of sample plates, the collection of plate digital images and the visual assessment of plate surface finish quality.

\section{References}

1. F Mirjalili, S Moradian and F Ameri, 'A new approach to investigate relationships between certain instrumentallymeasured appearance parameters and their visually-perceived equivalents in the automotive industry', Journal of Coatings Technology and Research, Vol 11, No 3, pp 341-350, 2014.

2. J M Parker, 'A robust machine vision system design to facilitate the automation of surface appearance inspections', Proceedings of the IEEE/ASME International Conference on Advanced Intelligent Mechatronics, Como, Italy, pp 87-92, 8-12 July 2001.

3. G Palardy, P Hubert, M Haider and L Lessard, 'Optimisation of RTM processing parameters for Class A surface finish', Composites Part B: Engineering, Vol 39, No 7-8, pp 1280-1286, 2008.

4. P J Schubel, N A Warrior, K N Kendall and C D Rudd, 'Characterisation of thermoset laminates for cosmetic automotive applications: Part I - Surface characterisation', Composites Part A: Applied Science and Manufacturing, Vol 37, No 10, pp 1734-1746, 2006.

5. S Hu, S V Hoa and R Ganesan, 'A new evaluation method for surface finish of composite automotive panels using waveform analysis', Journal of Composite Materials, Vol 43, No 8, pp 843 875, 2009.

6. E Summerscales, 'The visual assessment of finish', Production Engineers Journal, Vol 38, No 11, pp 637-640, 1959.

7. J M Parker, C Yew Lim, P Gnanaprakasam, H Zhen and J Istre, 'Inspection technology to facilitate automated quality control of highly specular, smooth coated surfaces', Proceedings of the IEEE International Conference on Robotics and Automation, Washington DC, USA, pp 2567-2574, 11-15 May 2002.

8. S Pal, S K Ghatak, S De and S DasGupta, 'Evaluation of surface roughness of a plasma-treated polymeric membrane by wavelet analysis and quantification of its enhanced performance', Applied Surface Science, Vol 255, No 5, Part 1, pp 2504-2511, 2008.

9. J G Daugman, 'Two-dimensional spectral analysis of cortical receptive field profiles', Vision Research, Vol 20, No 10, pp 847856, 1980.

10. A K Jain, F Farrokhnia and D H Alman, 'Texture analysis of automotive finishes', Proceedings of the SME Machine Vision Applications Conference, Detroit, USA, pp 1-16, 12-15 November 1990.

11. T Lindblad and J M Kinser, 'Inherent features of wavelets and pulse coupled networks', IEEE Transactions on Neural Networks, Vol 10, No 3, pp 607-614, 1999.

12. P Bojarczak, 'Visual algorithms for automatic detection of squat flaws in railway rails', Insight: Non-Destructive Testing and Condition Monitoring, Vol 55, No 7, pp 353-359, 2013.

13. A Movafeghi, M H Kargarnovin, H Soltanian-Zadeh, M Taheri, F Ghasemi, B Rokrok, K Edalati and N Rastkhah, 'Flaw detection improvement of digitised radiographs by morphological transformations', Insight: Non-Destructive Testing and Condition Monitoring, Vol 47, No 10, pp 625-630, 2005.

14. C M Leavey, M N James, J Summerscales and R Sutton, 'Damage detection in CFRP using wavelet scale correlation', Materials Science Forum, Vol 440-441, pp 211-218, 2003.

15. P Podsiadlo and G Stachowiak, 'Multi-scale representation of tribological surfaces', Proceedings of the Institution of Mechanical Engineers, Part J: Journal of Engineering Tribology, Vol 216, No 6, pp 463-479, 2002. 
16. M H Bharati and J F MacGregor, 'Image texture analysis: methods and comparisons', Chemometrics and Intelligent Laboratory Systems, Vol 72, No 1, pp 57-71, 2004.

17. S Palmer and W Hall, 'Surface evaluation of carbon fibre composites using wavelet texture analysis', Composites Part B: Engineering, Vol 43, No 2, pp 621-626, 2012.

18. S Palmer and W Hall, 'Objective surface evaluation of fibrereinforced polymer composites', Applied Composite Materials, Vol 20, No 4, pp 627-637, 2013.

19. S Palmer and W Hall, 'Evaluation of the robustness of surface characterisation of carbon fibre composites using wavelet texture analysis', Materials Science Forum, Vol 773-774, pp 234241, 2014.

20. Q Labrosse, C P Hoppins and J Summerscales, 'Objective assessment of the surface quality of coated surfaces', Insight: Non-Destructive Testing and Condition Monitoring, Vol 53, No 1, pp 16-20, 2011.

21. The MathWorks Inc, Matlab, Version 7.11.0.584 (R2010b), 2010.

22. The MathWorks Inc, Matlab Wavelet Toolbox, Version 4.6, 2010.

23. D B Percival and A T Walden, Wavelet Methods for Time Series Analysis, Cambridge University Press, Cambridge, UK, 2000.

24. J Zhang, X Wang and S Palmer, 'Performance of an objective fabric pilling evaluation method', Textile Research Journal, Vol 80, No 16, pp 1648-1657, 2010.

\section{Published by The British Institute of Non-Destructive Testing}

\section{Non-Destructive Testing $-2^{\text {nd }}$ Edition}

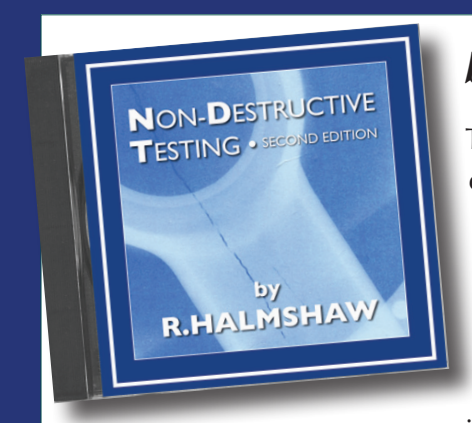

\section{by Dr R Halmshaw}

This edition was extensively revised to address recent advances in NDT technology. It covers all major aspects of NDT with a clear, practical approach. There is emphasis on applications and their relative importance. The Second Edition was produced in 1991 and published by Edward Arnold. This CD-ROM version, produced in 2004 from the 2nd Edition, has been reformatted and compiled by Dr Robin Shipp on behalf of The British Institute of Non-Destructive Testing.

ISBN: CD-ROM: 0903132354

Softcover printed edition: 0340545316

Price: BINDT Members: $£ 35$ + VAT; Non-Members: $£ 40$ + VAT

Available from: The British Institute of Non-Destructive Testing,

Newton Building, St George's Avenue, Northampton NN2 6JB, UK.

Tel: +44 (0)1604 89 3811; Fax: +44 (0)1604 89 3861; Email: info@bindt.org

Order online via the BINDT Bookstore at www.bindt.org/shopbindt

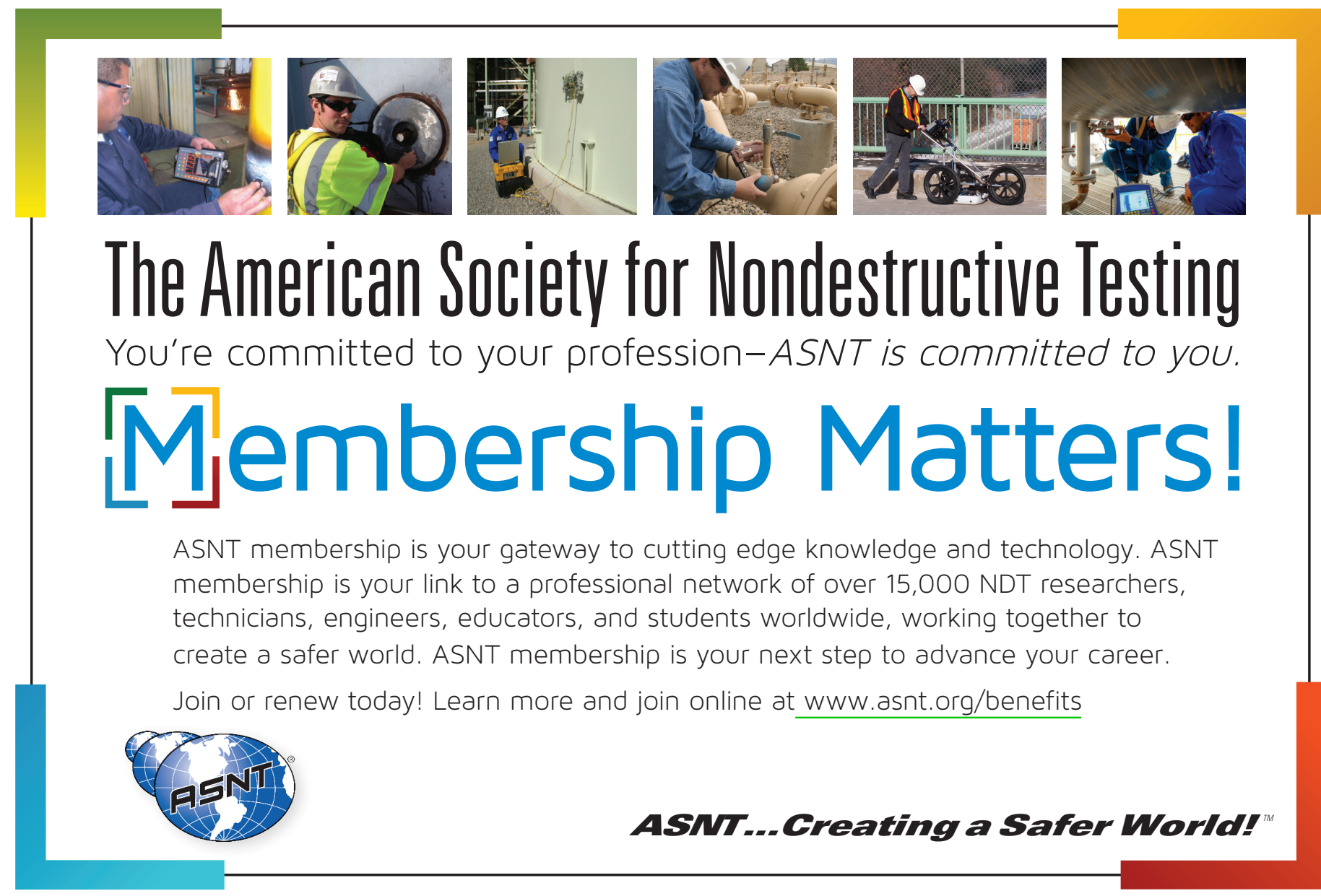

